\title{
АВТОМАТИЗАЦИЯ АЛГОРИТМА ИДЕНТИФИКАЦИИ НАИБОЛЕЕ ПЕРСПЕКТИВНЫХ ИННОВАЦИОННЫХ ИССЛЕДОВАТЕЛЬСКИХ НАПРАВЛЕНИЙ В СФЕРЕ АППАРАТНОГО РАЗВИТИЯ МЕДИЦИНЫ
}

\author{
(c) 2020 Ожгихин Иван Владимирович \\ Высшая инженерно-экономическая школа \\ Санкт-Петербургский политехнический университет Петра Великого (СПбПУ), \\ Россия, Санкт-Петербург \\ E-mail: mail@shvabe.com
}

() 2020 Рудская Ирина Андреевна

доктор экономических наук, Высшая инженерно-экономическая школа

Санкт-Петербургский политехнический университет Петра Великого (СПбПУ), Россия, Санкт-Петербург

E-mail: rudskaya_ia@spbstu.ru

Данная статья является продолжением статьи «Алгоритм идентификации наиболее перспективных инновационных исследовательских направлений в сфере аппаратного развития медицины», опубликованной в номере 189 журнала «Экономические науки» в 2020 году. В рамках предшествующей статьи был предложен соответствующий алгоритм, основанный на экстраполяции свойств цикла Гартнер. В рамках данной статьи реализуется последовательная автоматизация данного алгоритма, посредством высокоуровневого языка программирования Python. Представленный в рамках данной статьи программный код может быть заимствован, и использован для решения смежных задач.

Ключевые слова: питон, аппаратное развитие медицины, инновационные исследования, медицинское оборудование, лексема.

В статье [1] автором был предложен алгоритм идентификации наиболее перспективных инновационных исследовательских направлений в сфере аппаратного развития медицины. Одним из определяющих преимуществ данного алгоритма является возможность его автоматизации. В рамках данной статьи авторы последовательно детализируют специфику реализации каждого из выделенных этапов алгоритма, и формируют программный код, который может быть полностью или частично заимствован и применен для решения смежных задач. При этом, необходимо отметить, что под аппаратным развитием медицины понимается совокупность научно-исследовательских и опытноконструкторских работ, результатом которых являются как инновационное медицинское оборудование, так и инновационные решения в сфере автоматизации медицинской деятельности. Специфика развития данной области, как научно-исследовательская, так и рыночная, также подробно рассматриваются в статье [1].

Разработанный алгоритм дифференцирован на три ключевых этапа реализации. В первую очередь рассмотрим подготовительный этап алгоритма. В начале данного этапа подразумевается выбор источников информации. Так как предметом анализа является информационное поле исследований в области медицины, базовым ресурсом может выступать агрегатор (концентратор) результатов исследований. В сети интернет существует множество подобных агрегаторов, в частности: международные реферативные базы Scopus и Web of Science, электронные библиотеки Sciencedirect и Elibrary и многие другие. Однако, наиболее подходящей для целей автоматизированного исследования информационного поля является научная социальная сеть ResearchGate. Данное утверждение базируется на следующих свойствах данного ресурса:

1. Ha ResearchGate может индексироваться практически любая исследовательская работа, опубликованная где-либо. В следствии данного факта на ResearchGate сконцентрированы результаты исследований, представленные практически во всех упомянутых ранее базах данных.

2. ResearchGate не требует дополнительного рецензирования размещаемых работ и 
позволяет свободно вести дискуссию в комментариях к каждой из них. В следствии данного факта можно также выявлять реакцию научнопрофессионального сообщества на те или иные исследования.

3. ResearchGate не требует обязательной регистрации и позволяет осуществлять поиск вне аутентификации. Следовательно, автоматизированные алгоритмы поиска и сбора информации могут относительно стабильно взаимодействовать с ResearchGate. Безусловно, на сайте существует базовая информационная защита, не позволяющая открыто собирать информацию при помощи простейших автоматизированных алгоритмов, однако обход данной защиты является базовым и рассматривается далее.

Таким образом ResearchGate является наиболее эффективным источником информации, описывающем исследуемое информационное поле. В рамках завершения подготовительного этапа необходимо уточнить источники информации. B ResearchGate реализована система тегов, каждый из которых объединяет определённую область исследований. В рамках исследований медицинского характера, можно выделить следующие теги, присутствующие на ResearchGate: Medicine, Disease. Данная совокупность тегов является обще-информационной и включает в себя подавляющее большинство исследований определяющих область исследований. Безусловно, в рамках практической реализации приведенного алгоритма данный список может дополняться и уточнятся, однако на этапе теоретической отработки данная совокупность тегов полностью покрывает потребности в ресурсах описания информационного поля.

Далее рассмотрим исследовательский этап приведенного алгоритма. Данный этап может быть полностью автоматизирован. Для целей автоматизации данного этапа был определен высокоуровневый язык программирования Python. В начале реализации программного алгоритма производится сбор первичной информации. Единицей первичной информации выступает название конкретного исследования, содержащегося в базе данных в рамках рассматриваемых тегов. По соответствующим тегам ResearchGate выводит максимальное количество информации - 20 страниц по 100 записей, что суммарно дает 2000 наименований исследований по каждому из тегов. Процесс автоматизированного сбора информации в сети интернет называется «парсинг». Данный процесс можно подразделить на несколько этапов:

1. Отправка URL запроса. В данном случае базовыми запросами являются:

a. «https://www.researchgate.net/topic/ Disease/publications»

b. «https://www.researchgate.net/topic/ Medicine/publications»

2. Получение и сохраннее HTML кода соответствующей страницы.

3. Расшифровка CSS разметки сохраненных страниц.

4. Поиск по тегам необходимой информации. Наименования исследований сохранены в теге 'div' и классе - 'nova-e-text nova-e-text size-l nova-e-text - family-sans-serif nova-etext - spacing-none nova-e-text - color-inherit nova-v-publication-item_title'.

5. Сохранение необходимой информации (в данном случае наименований исследований) в порядковый список.

6. Удаление сопутствующей информации (элементов разметки, ссылок на ресурсы и т.д.) из элементов списка.

7. Переход на следующую порядковую страницу и повторение сформированной последовательности действий.

Каждая их приведенных задач может быть решена посредствам определённого инструмента. Так как на ResearchGate установлена базовая защита от автоматизированного сбора информации, в процессе отправки URL запроса необходимо смоделировать поведение человека. Для данных целей может быть использован инструмент из библиотеки selenium - webdriver. При этом необходимо соблюдать принцип рандомизации задержки, в соответствии с которым переведение человека в сети (переход по ссылкам, отправка запросов и т.д.) не может быть условно-постоянной во времени. Для целей формирования задержек предлагается использовать инструмент sleep из библиотеки time, а для целей рандомизации предлагается использовать инструмент uniform из библиотеки random. Эмпирически было определено, что необходимый диапазон рандомизации времени задержек для анализируемого ресурса составляет от 5.95 секунд до 9.15 секунд. Для целей расшифровки полученного HTML кода и последующей очистки результатов предлагается использовать библиотеки bs4 и re. Описанный алгоритм парсинга ResearchGate может быть приставлен в виде 
восьми последовательных этапов, представленных на рисунке 1.

В случае обработки иных тегов производится замена соответствующего URL в строке 3.1. Далее, в соответствии с предложенным ранее алгоритмом производится распределение первичной информации во времени. Так как каждое исследование в соответствии с архитектурой ResearchGate имеет дату опубликования в формате «Месяц-Год», данных этап также может быть автоматизирован путем интеграции в при- ведённых на рисунке 1 алгоритм следующего кода между этапами 7 и 8:

1. dates = usefull_inf.findAll('li', \{'class':'novae-list_item nova-v-publication-item_meta-dataitem'\})

2. for date in dates:

a. date_text $=$ date.text

b. try_list.append(date_text)

На следующем этапе предложенного алгоритма производится приведение первичной информации к единообразному виду. В данном случае,
1. Импорт необходимых библиотек

2. Открытие браузера

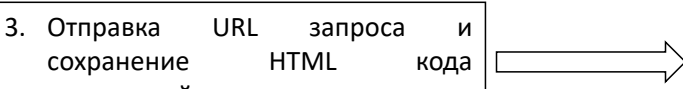
полученной страницы

4. Создание счетчика и списков для хранения данных

5. Создание цикла обработки

6. Поиск и сохранение названий исследований

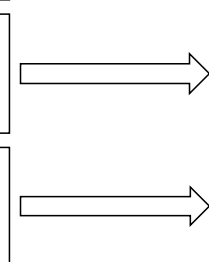

$\sqrt{3 .}$

1. $x=1$

2. main_list $=[]$

3. try_list $=[]$

1. while $x<=20$ :

Дальнейший код реализуется внутри цикла

1. usefull_inf =BeautifulSoup(response)

2. titles = usefull_inf.findAll('div', \{'class':'nova-e-text nova-e-text--size-I nova-e-text--family-sans-serif nova-e-text--spacing-none nova-e-text-color-inherit nova-v-publication-item__title'\})

7. Удаление метоинформации из названий исследований

1. for title in titles:

1. title_text $=$ re.sub $\left(r^{\prime}\left|\left\langle{ }^{\wedge}>\right]^{*}\right|>^{\prime}\right.$, ", $\operatorname{str}($ title $\left.)\right)$

2. main_list.append(title_text)

1. $x+=1$

2. if $x<=11$ :

1. button = browser.find_element_by_xpath $(1 /)^{*}[@ i d=" l i t e-$ page"]/main/section[3]/div/div[2]/div/div[101]/div/div/div/div[1]/d iv/div[12]/a')

2. button.click()

3. time.sleep(rd.uniform(5.95, 9.15))

4. response $=$ browser.page_source

3. else:

1. button = browser.find_element_by_xpath('//*[@id="litepage"]/main/section[3]/div/div[2]/div/div[101]/div/div/div/div[1]/d iv/div[13]/a')

2. button.click()

3. time.sleep(rd.uniform(5.95, 9.15))

4. response $=$ browser.page_source

Рисунок 1. Алгоритм парсинга первичной информации для тега Disease на ResearchGate, с использованием языка программирования Python 
информация структурно единообразна. Однако, надо отметить, что ResearchGate является международным ресурсов, в связи с чем многие исследования представлены на разных языках. В качестве базового языка предлагается использовать английский язык. Для целей автоматизированного перевода предлагается использовать инструмент Translator из библиотеки googletrans. Данный инструмент позволяет автоматически отправлять запросы в переводчик от Google и расшифровывать получаемые ответы. На рисунке 2 представлен алгоритм автоматизированного перевода анализируемой информации.

На следующем этапе предложенного алгоритма производится выделение лексем. Данная процедура является лингвистической и может быть подразделена несколько этапов, а именно:

1. Разделение базовых элементов (в данном случае названий исследований) на токены элементарные лексемы.

2. Лемматизация выделенных токенов. Данная процедура подразумевает приведение токенов к единой словарной форме, для целей исключения смысловых дублирований. Полученный список токенов уже можно охарактеризовать как список лексем.

3. Исключение лексем с низким смысловым наполнением - цифр, знаков пунктуации, предлогов и т.д.

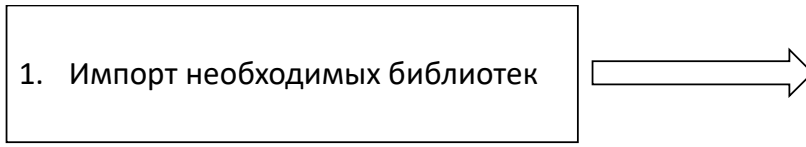

1. from googletrans import Translator

2. translator $=$ Translator ()

\section{1. trans_main_list $=[]$}

1. for sentens in main_list::

Дальнейший код реализуется внутри цикла

1. time.sleep(rd.uniform $(0.08,0.35))$

2. language $=$ translator.detect(sentens)

1. if language != 'en':

1. new_sentens = translator.translate(sentens)

2. trans_main_list.append(new_sentens.text)

2. else:

1. trans_main_list.append(sentens)

Рисунок 2. Алгоритм автоматизированного перевода анализируемой информации 


\begin{tabular}{|c|c|c|}
\hline 1. Импорт необходимых библиотек & 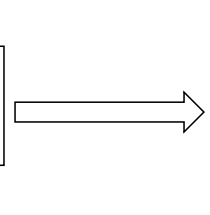 & $\begin{array}{l}\text { 1. import re } \\
\text { 2. from nltk import pos_tag } \\
\text { 3. from nltk.tokenize import word_tokenize } \\
\text { 4. from nltk.stem.snowball import SnowballStemmer } \\
\text { 5. from nltk.stem import WordNetLemmatizer } \\
\text { 6. lemmatizer = WordNetLemmatizer() }\end{array}$ \\
\hline $\begin{array}{l}\text { 2. Формирование списков для } \\
\begin{array}{l}\text { сохранения } \\
\text { результатов }\end{array}\end{array}$ & $\longrightarrow$ & $\begin{array}{l}\text { 1. } \text { blok_of_tokens }=[] \\
\text { 2. } \text { blok_of_sentens }=[] \\
\text { 3. } \text { blok_of_sentens_2 = [] }\end{array}$ \\
\hline $\begin{array}{l}\text { 3. Создание цикла обработки } \\
\text { названий статей }\end{array}$ & & $\begin{array}{l}\text { 1. for sentens in trans_main_list: } \\
\text { Дальнейший код реализуется внутри цикла до } 7 \text { этапа (включительно) }\end{array}$ \\
\hline $\begin{array}{l}\text { 4. Разделение названий статей на } \\
\text { токены }\end{array}$ & & 1. tokens $=$ word_tokenize(sentens) \\
\hline $\begin{array}{l}\text { 5. Лемматизация выделенных } \\
\text { токенов }\end{array}$ & $\longrightarrow$ & 1. tokens_2 $=\left[\right.$ lemmatizer.lemmatize $\left(\right.$ word, pos $\left.={ }^{\prime} v '\right)$ for word in tokens] \\
\hline $\begin{array}{l}\text { 6. Исключение лексем с низким } \\
\text { смысловым наполнением }\end{array}$ & $\longrightarrow$ & 1. tokens_3 $=\left[\right.$ re.sub $\left(r^{\prime}|W /| d / \mid s^{\prime}, "\right.$ ", word $)$ for word in tokens_2] \\
\hline $\begin{array}{l}\text { 7. Создание промежуточного списка } \\
\text { лексем }\end{array}$ & & $\begin{array}{l}\text { 1. tokens_4 = [] } \\
\text { 2. for } w \text { in tokens_3: } \\
\text { 1. if } w !=" \text { : } \\
\text { 1. tokens_4.append }(w) \\
\text { 3. blok_of_sentens.append(tokens_4) }\end{array}$ \\
\hline 8. Создание единого массива лексем & $\longrightarrow$ & $\begin{array}{l}\text { 1. for sentens in blok_of_sentens: } \\
\text { 1. full_sentense }={ }^{\prime} \text { '.join(sentens) } \\
\text { 2. blok_of_sentens_2.append(full_sentense) }\end{array}$ \\
\hline
\end{tabular}

Рисунок 3. Алгоритм формирования массива лексем

ность от 1 до n, где n - это число месяцев. На рисунке 4 представлен алгоритм формирования аналитического датафрейма.

На следующем этапе алгоритма для каждой из лексем строится модель регрессии. Для построения модели регрессии предлагается использовать метод наименьших квадратов. Однако, применение данного метода возможно исключительно для построения модели линейно регрессии, в то время как для целей данного исследования необходимо аппроксимировать данные экспоненциальной функцией. В случае если экспериментальная зависимость имеет нелинейный характер, путем замены переменных ее можно привести к линейному. Данный прием называется линеаризацией функциональных зависимостей [2]. Линеаризация экспоненциальной функциональной зависимости представлена на рисунке 5.

В соответствии с данным правилом необходимо преобразовать частоту в ее натураль- ный логарифм. Избавится от нулевых значений предлагается смещением частоты на единицу. Преобразовать сформированный датафрейм можно посредствам использования встроенной функции в библиотеке numpy, а именно:

analitic_data $=n p \cdot \log (($ year_day_mean +1$))$

На основе сформированного датафрейма могут быть построены соответствующие модели регрессии для каждой из выделенных лексем. Для данных целей используется библиотека statsmodels.api. Так как единственным анализируемым параметром получаемых моделей является коэффициент детерминации, целесообразно рассчитывать его сразу для каждой модели и сохранять в соответствующий список. На рисунке 6 представлен алгоритм построения моделей регрессии для выявленных лексем.

В завершении второго этапа предлагаемого алгоритма необходимо сформировать результирующий датафрейм, состоящий из структурированного списка токенов и соответствующих им 


\begin{tabular}{|c|c|c|}
\hline 1. Импорт необходимых библиотек & $\longrightarrow$ & $\begin{array}{l}\text { 1. import numpy as } n p \\
\text { 2. import pandas as } p d \\
\text { 3. from datetime import datetime } \\
\text { 4. from sklearn.feature_extraction.text import CountVectorizer } \\
\text { 5. count = CountVectorizer() }\end{array}$ \\
\hline 2. Формирование списка лексем & $\longrightarrow$ & 1. anal_data $=n p$.array(blok_of_sentens_2) \\
\hline $\begin{array}{l}\text { 3. Расчет частоты упоминания } \\
\text { лексем и формирование списков }\end{array}$ & $\longrightarrow$ & $\begin{array}{l}\text { 1. dag_of_words = count.fit_transform(anal_data) } \\
\text { 2. anal_array }=\text { dag_of_words.toarray() } \\
\text { 3. coll_names }=\text { count.get_feature_names() }\end{array}$ \\
\hline 4. Формирование датафрейма & $\longrightarrow$ & 1. data $=$ pd.DataFrame(anal_array, columns=coll_names)) \\
\hline $\begin{array}{l}\text { 5. Трансформация индексов строк } \\
\text { датафрейма в даты }\end{array}$ & $\longrightarrow$ & 1. data.index=pd.to_datetime(try_list[0:Ien(trans_main_list)]) \\
\hline $\begin{array}{l}\text { 6. Формирование мультииндекса и } \\
\text { группировка данных по месяцам }\end{array}$ & $\longrightarrow$ & $\begin{array}{l}\text { 1. data['Year']=data.index.year } \\
\text { 2. data['Mon']=data.index.month } \\
\text { 3. dates=data[['Year','Mon']] } \\
\text { 4. data.index=pd.Multilndex.from_tuples(dates.values.tolist(), names=dates.columns) } \\
\text { 5. year_day_mean=data.groupby(level=[0,1]).sum() } \\
\text { 6. year_day_mean.drop(['Year','Mon'],inplace=True,axis=1) }\end{array}$ \\
\hline
\end{tabular}

Рисунок 4. Алгоритм формирования аналитического датафрейма

\begin{tabular}{c}
$\begin{array}{c}\text { Исходный вид функции } \\
Y=a * e^{b * x}\end{array} \Rightarrow \begin{array}{c}\text { Преобразованная функция } \\
\ln Y=\ln a+b * x\end{array}$ \\
\hline
\end{tabular}

Рисунок 5. Линеаризация экспоненциальной функциональной зависимости

\begin{tabular}{|c|c|c|}
\hline 1. Импорт необходимых библиотек & $\longrightarrow$ & 1. import statsmodels.api as sm \\
\hline $\begin{array}{l}\text { 2. Формирование ряда независимых } \\
\text { переменных }\end{array}$ & $\longrightarrow$ & 1. $X=$ list $($ range $(1$, len $($ analitic_data $)+1))$ \\
\hline $\begin{array}{l}\text { 3. Формирование рядов зависимых } \\
\text { переменных }\end{array}$ & $\longrightarrow$ & 1. $Y_{-}$list $=$analitic_data.columns \\
\hline $\begin{array}{l}\text { 4. Создание списка для сохранения } \\
\text { коэффициентов детерминации } \\
\text { моделей }\end{array}$ & $\square$ & 1. models_list $=[]$ \\
\hline $\begin{array}{l}\text { 5. Создание цикла для перебора } \\
\text { лексем }\end{array}$ & $\square$ & $\begin{array}{l}\text { 1. for tok in Y_list: } \\
\text { Дальнейший код реализуется внутри цикла }\end{array}$ \\
\hline $\begin{array}{l}\text { 6. Выбор ряда зависимой } \\
\text { переменной }\end{array}$ & $\longrightarrow$ & 1. $y=$ analitic_data[tok] \\
\hline 7. Формирование константы & $\longrightarrow$ & 1. $X=$ sm.add_constant $(X)$ \\
\hline 8. Построение модели регрессии & $\longrightarrow$ & 1. odel $=\operatorname{sm} \cdot \mathrm{OLS}(\mathrm{y}, X) \cdot f i t())$ \\
\hline 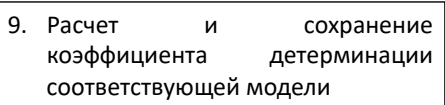 & $\longrightarrow$ & 1. models_list.append(model.rsquared) \\
\hline
\end{tabular}


коэффициентов детерминации. Для решения данной задачи предлагается использовать библиотеку csv, позволяющую сохранить сформированный датафрейм в файле соответствующего формата. На рисунке 7 представлен алгоритм формирования и сохранения результирующего датафрейма.

Полученный по результатам исследовательского этапа алгоритма, датафрейм содержит необходимый и достаточный объем информации для целей дальнейшего анализа. В рамках завершающего - аналитического этапа алгоритма производится последовательная ментальная обработка полученного результата. В первую очередь проводится первичный отбор лексем в соответствии с коэффициентом детерминации. В соответствии с шкалой Чедока, минимально допустимым значением коэффициента детерминации является 0,5. Следовательно, на данном этапе из массива исключаются все лексемы, коэффициент детерминации моделей которых составляет менее данного пограничного значения.

На следующем этапе анализа производится метаосмысление выделенных лексем. В данном случае каждая из лексем осмысляется в контексте исследований в области разработки медицинского оборудования. На данном этапе лексемы могут быть разделены на две базовые группы:

1. Условно осмысляемые в контексте исследований в области разработки медицинского оборудования. В данную группу входят лексемы как непосредственно связанные с исследованиями в области разработки медицинского обору- дования, так и опосредованно.

2. Условно не осмысляемые в контексте исследований в области разработки медицинского оборудования. Данная группа включает в себя отстраненные или полностью контекстуально не связанные с исследованиями в области разработки медицинского оборудования лексемы, так и общие лексемы, которые могут быть связаны практически с любой исследовательской областью.

Полученный по результатам данной обработки массив лексем является базисом идентификации наиболее перспективных исследовательских направлений в сфере аппаратного развития медицины. Для целей формирование массива наиболее значимых лексем и исследовательских тезисов необходимо построить ассоциативную карту, объединяющую все выделенных лексемы в исследовательские области. Данные комплексные лексемы являются исследовательскими тезисами, на основе которых в дальнейшем производится формирование комплекса наиболее перспективных исследовательских направлений.

Таким образом, предложенный алгоритм использует в качестве исследовательского базиса объективную информацию, что позволяет избежать субъективности аналитических результатов. Так как часть алгоритма полностью автоматизирована, он может использовать включительно специалистами в предметной области, не владеющими специализированными знаниями в области статистики и анализа данных.

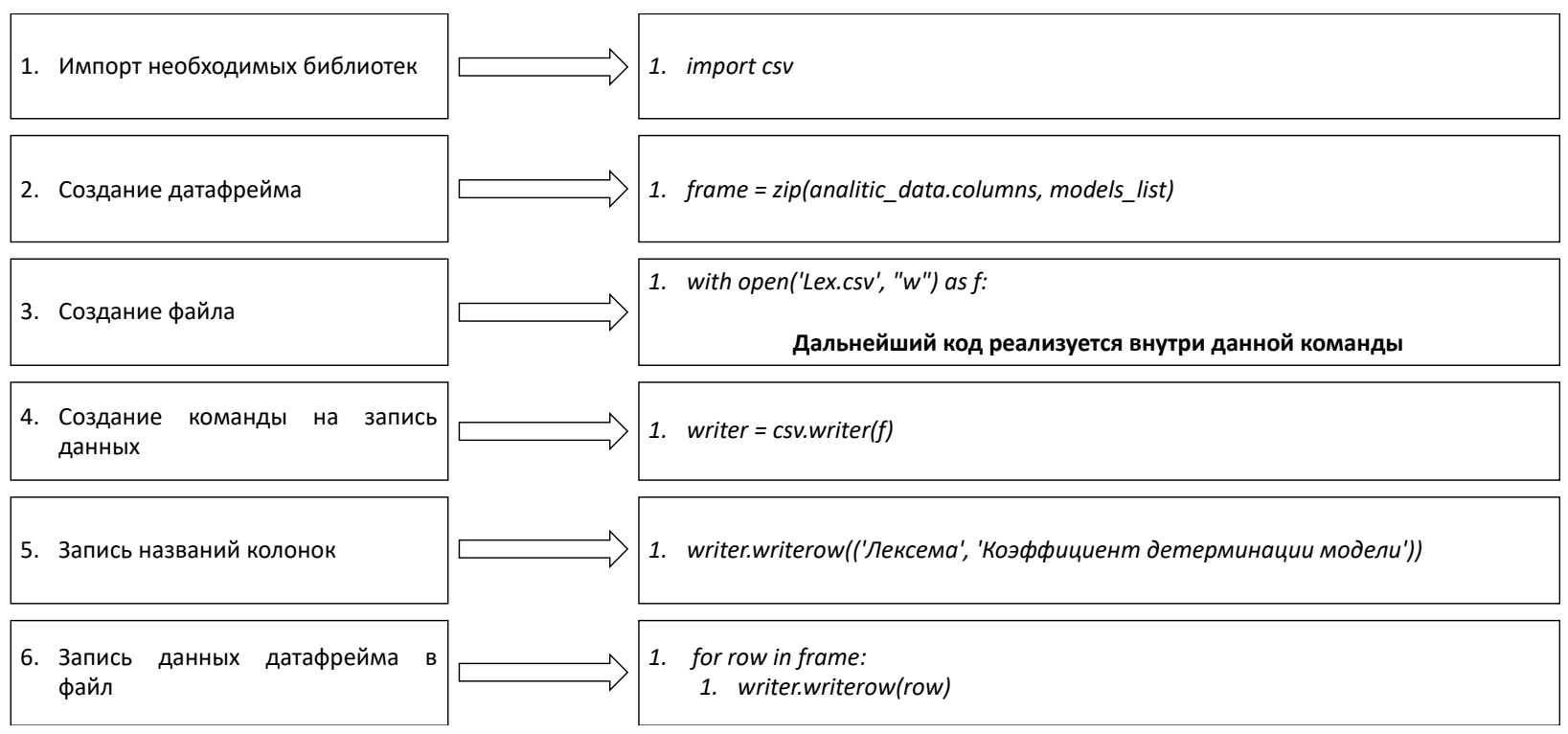

Рисунок 7. Алгоритм формирования и сохранения результирующего датафрейма 


\section{Библиографический список}

1. Ожгихин И.В., Рудская И.А. Алгоритм идентификации наиболее перспективных инновационных исследовательских направлений в сфере аппаратного развития медицины // Экономические науки. 2020. № 189. С. 69-74.

2. Линеаризация функциональных зависимостей // URL: https://mykonspekts.ru/1-31084.html

3. Седякина А.А., Конников Е.А. Методика оценки совместимости научно-исследовательского коллектива // Экономические науки. 2020. № 188. С. 77-87.

4. Родионов Д.Г., Мельниченко А. М. Моделирование организационно-экономического механизма управления развитием инновационной среды // Вестник Алтайской академии экономики и права. 2019. № 11-3. С. 72-83.

5. Konnikov E.A., Konnikova O. A. Methodology of Hype Monetization in the Internet Environment According to the Life Cycle Theory // ACM International Conference Proceeding Series. Conference: SPBPU IDE '19: International Scientific Conference on Innovations in Digital Economy 2019. 